\title{
Factors Affecting Clinical Results after Corrective Osteotomy for Lumbar Degenerative Kyphosis
}

\author{
Whoan Jeang Kim¹, Jong Won Kang ${ }^{1}$, Sung II Kang ${ }^{1}$, Hwan II Sung ${ }^{1}$ \\ Kun Young Park², Jae Guk Park ${ }^{3}$, Won Cho Kwon', Won Sik Choy ${ }^{1}$ \\ 'Department of Orthopedic Surgery, Eulji University College of Medicine, Daejeon, Korea \\ ${ }^{2}$ Daejeon Veterans Hospital, Daejeon, Korea \\ ${ }^{3}$ Hongseong Medical Center, Hongseong, Korea
}

Study Design: This study is a prospective, clinical study for lumbar degenerative kyphosis.

Purpose: To determine the factors affecting postoperative clinical outcomes in patients who undergo corrective osteotomy for lumbar degenerative kyphosis.

Overview of Literature: Only a small number of studies have reported clinical results for surgery for lumbar degenerative kyphosis. There are almost no studies about prognostic factors that predict postoperative clinical results.

Methods: This study involved 25 patients who were diagnosed with lumbar degenerative kyphosis and who underwent corrective osteotomy following gait analysis. A pedicle subtraction osteotomy was done at the third lumbar vertebra (L 3). Regarding the fusion level, surgery was done within a range from T10 proximally to S1 distally. Of these, for rigid fixation of a distal part, an iliac screw was used. Pain was evaluated using a 10-point pain scale and a questionnaire about activities. We also evaluated cosmesis and subjective satisfaction using a modified version of the Scoliosis Research Society Outcome22 (SRS-22) instrument. This assessment was done using a 5-point scale which was designed by us. We assigned patients to group A (good clinical outcomes) if their postoperative pain score was lower than 4 (of 10 points) and if scores indicating activity, cosmesis and subjective satisfaction were higher than 11 (of 15 points). All other patients were assigned to group B (poor clinical outcomes).

Results: Clinical outcomes were good in 64\% of patients (16/25) and poor in 36\% (9/25). Regarding cosmesis and subjective satisfaction, there were significant differences between the two groups. There were also significant differences in physical factors of individual patients such as body mass index (BMI): $23.78 \pm 2.79$ in group A and $26.44 \pm 2.75$ in group B. On gait analysis, there was a significant difference in the dynamic pelvic tilt: $7.5 \pm 3.3^{\circ}$ in group A and $11.72 \pm 1.89^{\circ}$ in group B.

Conclusions: There is no correlation between preoperative degree of kyphotic deformity and clinical outcomes. The degree of anterior rotation of pelvic tilt does not change significantly; rather, compensatory mechanisms of the pelvis and BMI were found to have more influence. Because neither the degree of satisfaction with clinical outcomes nor the increased activity was relatively higher, a more sincere decision should be made before recommending corrective osteotomy for degenerative lumbar kyphosis.

Key Words: Lumbar degenerative kyphosis, Dynamic pelvic tilt, Radiological assessment, BMI and clinical outcomes

Received Jul 23, 2009; 1st Revised Nov 30, 2009; Accepted Dec 1, 2009

Corresponding author: Jong Won Kang, MD

Department of Orthopaedic Surgery, Eulji University College of Medicine,

1306 Dunsan-dong, Seo-gu, Daejeon 302-799, Korea

Tel: +82-42-611-3279, Fax: +82-42-259-1289, E-mail: jwkang@eulji.ac.kr 


\section{Introduction}

Lumbar degenerative kyphosis is a characteristic disease that features dynamic stooping on gait analysis due to degeneration of the lumbar extensor muscle and the hip extensor muscle. The initiation of gait movement and dynamic stooping are unlike the static stooping seen in cases of senile kyphosis or ankylosing spondylitis. In recent years, as the number of elderly individuals in the population has been increasing, active treatments have been demanded for cases of lumbar degenerative kyphosis to improve quality of life. However, in most cases, patients demanding treatments are relatively old. It is highly probable that complications might occur because of a loss of the fixation due to a long-term surgery. Complications include bleeding, wound infection at the operation site and osteoporosis. General factors affecting clinical outcomes in the surgical correction of long level spinal deformity include incomplete correction, loss of fixation force due to fixation failure, and non-union. In lumbar degenerative kyphosis, there is a dynamic sagittal imbalance. Therefore, a lack of sufficient correction and a loss of the fixation angle can cause a restooping to appear, and this will affect clinical outcomes. Understanding the dynamic concept through gait analysis is more important. Also, despite the presence of a correction loss, there are some cases in which clinical outcomes are good. In contrast, lack of a sufficient correction and a loss of the correction angle causes some patients to complain of persistent pain following surgery, and these are patients whose degree of satisfaction with surgical outcomes is not relatively higher than their preoperative level of satisfaction with their condition. It is therefore likely to be another cause associated with clinical outcomes. In particular, we considered as candidates pain, loss of activity, external appearance, and subjective degree of satisfaction. Depending on the characteristics of individual patients, there might even be a great difference in these factor and this might substantially affect clinical outcomes. This highlights the difficulty in making a decision to do surgery.

To date, only a small number of studies have reported clinical results for surgery for lumbar degenerative kyphosis. There are almost no studies about prognostic factors that predict postoperative clinical results.

Given the above background, we measured physical factors of individual patients, including bone density, body mass index (BMI), the presence of underlying medical dis- ease, and pelvic tilt (indicating a sagittal imbalance), using preoperative gait analysis and radiography. Then, attempts were made to compare the degree of kyphosis based on (a) the Takemitsu classification, (b) trunk tilt based on gait analysis, (c) the degree of atrophy of the lumbar extensor muscle based on magnetic resonance imaging (MRI) scans, and (d) the degree of a loss of the postoperative correction angle as compared with the postoperative clinical outcomes.

\section{Materials and Methods}

Of 30 patients who underwent surgery between June 2004 and December 2007 following preoperative gait analysis for lumbar degenerative kyphosis, and excluding cases in which an osteoporotic compression fracture was present, we enrolled 25 that showed a past history of spine surgery (for degenerative disease of knee joint or hip joint) and for which data was available for a minimum of 18 months of follow-up. The mean follow-up period was 24.4 months (range, 18 to 39 months) and mean age was 66 years (range, 58 to 74 years). All were women.

For each patient, a pedicle subtraction osteotomy was done at the third lumbar vertebra (L 3). Regarding the fusion level, surgery was done within a range from T10 proximally to $\mathrm{S} 1$ distally. For the rigid fixation of a distal part in these patients, an iliac screw was used. For each patient, clinical results were evaluated using a questionnaire at preoperative and postoperative outpatient visits. Pain was evaluated using a 10-point pain scale (Table 1) [1]. We also administered a questionnaire study about physical activity, cosmesis and subjective satisfaction (Table 2). We conducted using a modified version of the Scoliosis Research Soci-

Table 1. Patient pain scale: self-evaluation

\begin{tabular}{lcc}
\hline \hline Type of pain & Preop & Postop \\
\hline No. of significant pain & 1 & 1 \\
Occasional pain & 2 & 2 \\
Activity related, relief with rest & 3 & 3 \\
Requiring occasional NSAIDs & 4 & 4 \\
Interferes with work, constant NSAIDs & 5 & 5 \\
Inferferes with work, NSAIDs, pain modlities & 6 & 6 \\
Requiring occasional opiates & 7 & 7 \\
Constant, regular opiate use & 8 & 8 \\
Constant, bed to chair activity only, opiates & 9 & 9 \\
Morphine pump, high dose constant opiates & 10 & 10 \\
\hline
\end{tabular}

Preop: Preoperation, Postop: Postoperation, NSAIDs: Nonsteroidal antiinflammatory drugs. 
ety Outcome-22 (SRS-22) [2]. This assessment was done using a 5-point scale which was designed by us. We assigned patients to group A (good clinical outcomes) if their postoperative pain score was lower than 4 (of 10 points) and if scores indicating activity, cosmesis and subjective satisfaction were higher than 11 (of 15 points). All other patients were assigned to group B (poor clinical outcomes). Physical factors of individual patients - bone densi-

Table 2. Clinical assessment

\begin{tabular}{llcc}
\hline \hline Scales & Items & Preop & Postop \\
\hline \multirow{2}{*}{ Activity } & Bedridden/wheelchair & 1 & 1 \\
& Primarily no activity & 2 & 2 \\
& Light labor, such as household chores & 3 & 3 \\
& Moderate manual labor and moderate sports, such as walking and biking & 4 & 5 \\
& Full activities without restriction & 1 & 1 \\
Cosmesis & Very bad & 2 & 3 \\
& Bad & Fair & 2 \\
& Good & 3 & 4 \\
& Very good & 4 & 5 \\
& Extremely dissatisfied & 5 & 1 \\
& Somewhat dissatisfied & 2 & 2 \\
& Neither satisfied nor dissatisfied & 3 & 3 \\
& Somewhat satisfied & 4 & 5 \\
& Extremely satisfied & 5 & 5 \\
\hline
\end{tabular}

Preop: Preoperation, Postop: Postoperation.

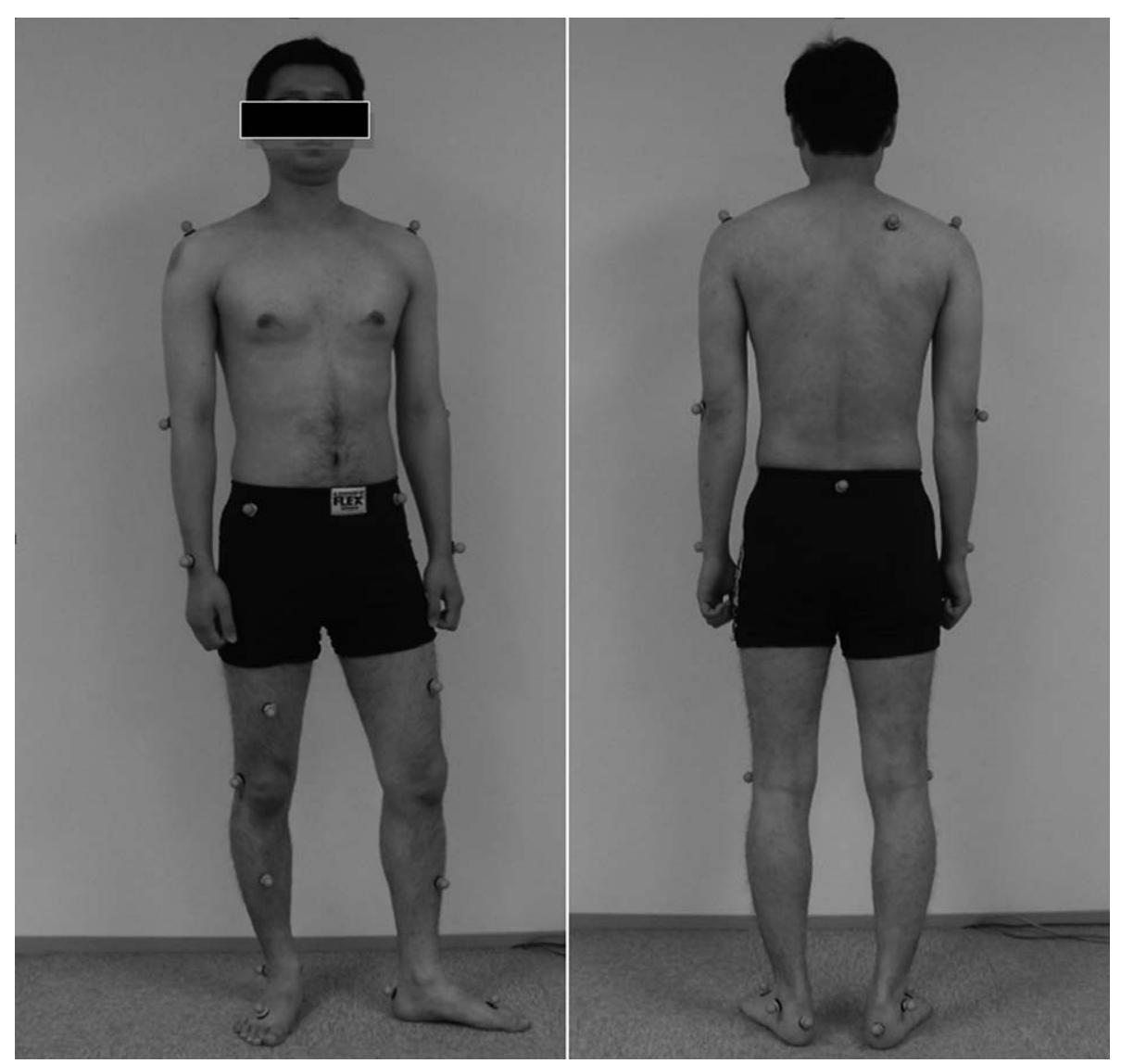

Fig. 1. Modified Helen-Hayes method. 
ty, BMI, the presence of underlying diseases, and pelvic tilt indicating a sagittal imbalance - were measured preoperatively using gait analysis and radiography. Attempts were then made to compare (A) the degree of kyphosis based on the classification system of Takemitsu, trunk tilt based on gait analysis, the degree of atrophy of the lumbar extensor muscle based on MRI scans, and extent of decrease of the postoperative correction angle with postoperative clinical outcomes.

A bone mineral density test was done using a Prodigy Advance instrument (General Electric, Milwaukee, WI, USA) for cases in which there was kyphotic deformity. Owing to this, and excluding the vertebral spine, we evaluated bone density, using the minimal value for the following bones: $\mathrm{T}$-scores of neck, wards triangle, trochanter and shaft of the proximal femur.

BMI was expressed as weight $(\mathrm{kg}) /$ height $(\mathrm{m})^{2}$ following the measurement of maximal height and weight prior to ambulatory movement.

For the preoperative gait analysis, we used an Eagle camera system and an AMTI power plate (force plate; Advanced Mechanical Technology Inc., Watertown, MA, USA). Data processing was done using Eva Real Time (EvaRT software ver. 4.2, Motion Analysis ${ }^{\circledR}$, Santa Rosa, CA, USA) and Orthotrak (Motion Analysis ${ }^{\circledR}$ software). A passive reflective marker was attached to the upper extremities, the lower extremities and the body trunk with the use of Modified Helen-Hayes methods (which are used the most frequently) (Fig. 1). During gait analysis, pelvic tilt was measured based on the slope value of a line connecting anterior superior iliac spine and posterior superior iliac spine. Measurements obtained prior to gait analysis were determined to be a static pelvic tilt. Thereafter, with the patient in a comfortable posture, ambulation analysis was done repeatedly for approximately five minutes. These three measurements were then averaged. The mean was defined as the dynamic pelvic tilt.

A radiologic examination was done for the anteroposterior and lateral images of the whole spine including the head and pelvis in a standing position for which both arms were placed on the shoulder and the knee joint was maximally extended. Pelvic tilt was obtained by measuring the angle formed by the vertical line crossing the hip axis of the overall lateral surface of the vertebra and the line connecting to the center of the $\mathrm{S} 1$ superior border at the mid-point connecting the center of the bilateral hip joints [3]. The degree of kyphosis was classified based on the radiological classifi- cation system of Takemitsu [4,5].

The degree of atrophy of the lumbar extensor muscle based on MRI scans was confirmed using the ratio between fat and muscle and between muscle and disc. Using a $\pi(\mathrm{Pi})$ view star (Infinit Technology, Meryland, CA, USA) for a T2-weighted axial image of the L4-5 disc space level, the paravertebral muscle was identified using an region of interest. Then, the relative ratio of paravertebral muscle to the disc area was obtained. Using a pseudo-color masking effect, the fat-containing ratio in the paravertebral muscles was obtained [3].

For measurement of a correction loss on the lateral view of the overall shape of the vertebral spine, which was acquired radiologically both preoperatively and at a final follow-up, Cobb's methods were used. The measurement was made at levels ranging from the most superior to the most inferior level of the vertebral spine.

Statistical analysis was done using SPSS ver. 13.0 (SPSS Inc., Chicago, IL, USA), for which an independent t-test, a paired t-test and a Mann-Whitney test were used.

\section{Results}

Clinical results were found to be good in 64\% (16/25) of cases and poor in 36\% (9/25). In group A, where clinical outcomes were found to be good, scores for each parameter were: pain $(2.121 \pm 05)$, the activity (3.4 \pm 0.18$)$, cosmesis $(4.24 \pm 0.22)$ and subjective satisfaction $(4.41 \pm 0.62)$. In group B, where clinical outcomes were poor, the corresponding scores were $2.89 \pm 1.45,3.11 \pm 0.87,2.78 \pm$ 0.97 and $1.78 \pm 0.83$ in the corresponding order. Regarding cosmesis and subjective satisfaction, there were significant differences between the two groups. Pain was reduced in both groups. But there was no significant difference in activity between the two groups or between preoperative and postoperative tests (Table 3).

Regarding physical factors, BMI was $23.78 \pm 2.79$ in group A and $26.44 \pm 2.75$ in group B. This difference reached statistical significance $(p=0.033)$. Bone density was $-2.56 \pm 0.76$ for group A and $-2.16 \pm 0.91$ for group B. Regarding underlying medical disease, diabetes mellitus was seen in 0 of 16 cases in group A and in one of nine cases in group B. Hypertension was seen in nine of 16 cases in group A and four of nine cases in group B. These differences were not significant (Tables 4 and 5).

On radiological assessment, preoperative pelvic tilt was 
Table 3. Clinical results

\begin{tabular}{llccc}
\hline \hline & & Group A (n=16) & Group B (n=9) & $p$-value \\
\hline \multirow{2}{*}{ Pain } & Preop & $6.06 \pm 0.9$ & $5.67 \pm 0.5$ & 0.24 \\
& Postop & $2.12 \pm 1.05$ & $2.89 \pm 1.45$ & 0.13 \\
\multirow{5}{*}{ Activity } & Improvement & $-3.94 \pm 1.64$ & $-2.78 \pm 1.48$ & 0.09 \\
& Preop & $2.91 \pm 0.47$ & $2.67 \pm 0.5$ & 0.85 \\
& Postop & $3.4 \pm 0.18$ & $3.11 \pm 0.87$ & 0.76 \\
Cosmesis & Improvement & $0.49 \pm 0.59$ & $0.44 \pm 1.13$ & 0.08 \\
& Preop & $2.41 \pm 0.71$ & $2.22 \pm 0.44$ & 0.48 \\
& Postop & $4.24 \pm 0.22$ & $2.78 \pm 0.97$ & 0.002 \\
Satisfaction & Improvement & $1.82 \pm 0.88$ & $0.56 \pm 1.01$ & 0.007 \\
\hline
\end{tabular}

Preop: Preoperation, Postop: Postoperation.

Table 4. Correlation factors of clinical results

\begin{tabular}{lccc}
\hline \hline & Group A (n=16) & Group B (n=9) & $p$-value \\
\hline Age & $67 \pm 4.97$ & $64 \pm 4.03$ & 0.133 \\
BMD & $-2.56 \pm 0.76$ & $-2.16 \pm 0.91$ & 0.25 \\
BMI & $23.78 \pm 2.79$ & $26.44 \pm 2.75$ & 0.033 \\
Radiologic pelvic tilt & $45.87 \pm 4.04$ & $46.5 \pm 7.5$ & 0.78 \\
Takemitsu type & $2.5 \pm 0.9$ & $2.4 \pm 0.7$ & 0.79 \\
Gait pelvic tilt (static) & $12.7 \pm 0.62$ & $12.4 \pm 1.55$ & 0.42 \\
Gait pelvic tilt (dynamic) & $7.5 \pm 3.3$ & $11.72 \pm 1.89$ & 0.03 \\
Gait pelvic tilt (S-D) & $4.8 \pm 3.25$ & $0.71 \pm 0.99$ & 0.006 \\
Gait trunk tilt & $20.24 \pm 13.6$ & $14.7 \pm 13.34$ & 0.41 \\
Fat-muscle ratio & $45.17 \pm 3.77$ & $46.44 \pm 3.81$ & 0.43 \\
Muscle-disc ratio & $0.96 \pm 0.06$ & $0.92 \pm 0.12$ & 0.37 \\
Correction loss & $6.68 \pm 4.93$ & $6.74 \pm 8.04$ & 0.97 \\
\hline
\end{tabular}

BMD: Bone mineral density, BMI: Body mass index.

Table 5. DM, HTN, screw loosening \& clinical results

\begin{tabular}{lcc}
\hline \hline & DM $(+)$ & DM (-) \\
\hline Group A & 0 & 16 \\
Group B & 1 & 8 \\
& $p=0.34$ & \\
\hline & HTN $(+)$ & HTN (-) \\
\hline Group A & 9 & 7 \\
Group B & 4 & 5 \\
& $p=0.42$ & \\
\hline & Loosening (+) & 14 \\
\hline Group A & 2 & 5 \\
Group B & 4 & Loosening (-) \\
& $p=0.15$ &
\end{tabular}

DM: Diabetes mellitus, HTN: Hypertension.

$45.8 \pm 4.0^{\circ}$ in group $\mathrm{A}$ and $46.5 \pm 7.5^{\circ}$ in group B. On gait analysis, static pelvic tilt was $12.7 \pm 0.62^{\circ}$ in group $\mathrm{A}$ and $12.42 \pm 1.55^{\circ}$ in group $B$. There were no significant differences in pelvic tilt in this series. On gait analysis, however, dynamic pelvic tilt was $7.5 \pm 3.3^{\circ}$ for group $\mathrm{A}$ and $11.72 \pm 1.89^{\circ}$ for group B. This difference reached statistical significance $(p=0.03)$.

The degree of kyphotic deformity based on the Takemitsu classification was found to be I: 2 , II: 6 , III: 7, IV: 1 in group A and II: 6, III: 2, IV: 1 in group B. On gait analysis, body trunk tilt was $20.24 \pm 13.6^{\circ}$ in group A and $14.7 \pm$ $13.34^{\circ}$ in group B. This difference did not reach statistical significance $(p=0.41)$.

The ratio of paravertebral muscle to the disc area indicates the degree of atrophy of the lumbar extensor muscles based on MRI scans; the ratio was $45.17 \pm 3.77 \%$ in group A and $46.44 \pm 3.81 \%$ in group B $(p=0.43)$. With regard to the ratio of paravertebral muscle to the disc area, it was 0.96 \pm 0.06 in group A and $0.92 \pm 0.12$ in group B. These differences did not reach statistical significance $(p=0.37)$.

The postoperative decrease in the correction angle was $6.684 .93^{\circ}$ in group A and 6.748.04 ${ }^{\circ}$ in group B. This difference did not reach statistical significance $(p=0.97)$. 


\section{Discussion}

Lumbar degenerative kyphosis is a common disease in the elderly. In recent years, as the number of individuals in the elderly population has been increasing, active treatments have been demanded by patients for cases of lumbar degenerative kyphosis in order to improve their quality of life. However, the average age at the time of surgery is relatively old. It is highly probable that complications will occur because of a loss of fixation force due to long level surgery, bleeding, wound infection at the surgical site or osteoporosis. In patients with a kyphotic deformity, an incomplete correction can achieve a sufficient correction through a pedicle subtraction osteotomy. We also [6] reported that a sufficient extent of sagittal correction can be obtained through an osteotomy using a pedicle. The reduction in correction angle occurs in the disc space where there is a mobile segment rather than an osteotomy site. It mainly occurs at the lower fixation of osteotomy site. To prevent this, the use of an anterior support has been reported to be desirable $[7,8]$. Also, during lumbar degenerative kyphosis where a long level fusion is needed, an Iliac screw is used. Thus, a stable fixation force is provided for a weak osteoporotic vertebra. This has been reported to reduce complications such as a reduction in correction angle, pseudoarthrosis, and screw loosening [9-11].

The pelvis plays a key role in the functioning of the vertebral spine. The importance of pelvic tilt in spino-pelvic balance has been emphasized. This is also the most important indicator based on the degree of balance or compensation of the pelvis that can be ascertained $[12,13]$. In patients with lumbar degenerative kyphosis in particular, posterior pelvic tilt occurs to compensate for stooping. This eventually leads to an increased pelvic angle. However, the vertebral deformity of lumbar degenerative kyphosis and the compensatory mechanism of the pelvis are diseases that develop when the skeletal deformity occurs due to a weakness in the lumbar extensor muscle. Different from ankylosing spondylitis or iatrogenic flat back syndrome, they reflect a dynamic imbalance in the sagittal plane. A radiogram cannot be solely used to clarify the correlations between vertebral spine, pelvis and lower extremities, which change at every time point during ambulation. It is therefore necessary to examine dynamic changes using gait analysis [14-16]. We also reported that gait analysis was the only method that can lead to the diagnosis of a dynamic imbalance of the sagittal plane in patients with lumbar degenerative kyphosis [17].

In our series, pelvic tilt on radiography was found to be $45.8 \pm 4.0^{\circ}$ in group A and $46.5 \pm 7.5^{\circ}$ in group B. On gait analysis, static pelvic tilt was found to be $12.7 \pm 0.62^{\circ}$ in group A and $12.42 \pm 1.55^{\circ}$ in group B. These differences did not reach statistical significance. During ambulation, however, dynamic pelvic tilt was $7.5 \pm 3.3^{\circ}$ in group $\mathrm{A}$ and $11.72 \pm 1.89^{\circ}$ in group B. This difference reached statistical significance. This might be because a posterior pelvic tilt triggered by a pelvic compensation mechanism was involved in group A and not in group B. Lee et al. [18] also reported that the most effective compensatory mechanism in resolving stooping in cases of lumbar degenerative kyphosis was posterior rotation of the pelvis. The most important muscles involved in posterior rotation of the pelvis included hip extensor muscles such as gluteus maximus and gluteus medius. As per guidelines for diagnosis and treatment, in patients who show a posterior pelvic tilt on a preoperative gait analysis, stooping is postoperatively improved. In patients who achieve an excessive extent of anterior pelvic tilt despite a lack of insufficient sagittal fixation or a loss of fixation force, one of the peculiar complications featuring persistent flexion of the waist is restooping. The reason is that the pelvic extensor muscle becomes fragile. The importance of gait analysis rather than a radiological assessment has been underscored. Due to a lack of definitive criteria for excessive anterior tilt, however, it is difficult to clinically apply a gait analysis. In the current study, in the group where the clinical outcomes were good on gait analysis, there were changes from a static pelvic tilt of $12.7 \pm 0.62^{\circ}$ to a dynamic pelvic tilt of $7.5 \pm 3.3^{\circ}$. This shows that there were changes in posterior pelvic tilt of 4.8 $\pm 3.25^{\circ}$. In the group where clinical outcomes were poor, there were changes from a static pelvic tilt of $12.42 \pm$ $1.55^{\circ}$ to a dynamic pelvic tilt of $11.72 \pm 1.89^{\circ}$. This indicated changes in posterior pelvic tilt of $0.71 \pm 0.99^{\circ}$. These results indicate that there were almost no changes in pelvic tilt angle during ambulation. Based on these results, it can be inferred that involvement of compensatory mechanisms for posterior pelvic tilt might be closely associated with clinical outcomes.

In addition, the degree of kyphotic deformity based on the Takemitsu classification was: I: 2, II: 6, III: 7, IV: 1 in group A and II: 6, III: 2, IV: 1 in group B. These results indicate that there were no significant correlations of the degree of kyphotic deformity based on the Takemitsu classification with clinical outcomes. Lumbar degenerative 
kyphosis is a characteristic disease featuring dynamic stooping where the waist is bent in proportion to the gait due to the degeneration of the lumbar extensor muscle and hip extensor muscle or because of the initiation of gait movement, unlike the static stooping seen in cases of senile kyphosis or ankylosing spondylitis. Radiologically, the degree of kyphotic deformity is the static factor. Regarding clinical outcomes of lumbar degenerative kyphosis, the dynamic factor is important.

In most elderly patients, due to the presence of osteoporosis, complications can occur because of the weakness of the fixation force. Accordingly, bone density level might affect clinical outcomes. In the current study, however, the bone density level was $-2.56 \pm 0.76$ in group A and $-2.16 \pm$ 0.91 in group B. In both groups, there were findings suggestive of osteoporosis. Due to the presence of severe osteoporosis, patients who had an osteoporotic multiple compression fracture were excluded. This might have diminished the effects on clinical outcomes in the current study. The correction angle was found to be $6.68 \pm 4.93^{\circ}$ in the group A and $6.74 \pm 8.04^{\circ}$ in the group B. In other words, there were no significant correlations with clinical outcomes. In addition to surgical factors, the degree of reduction of the correction angle, and screw loosenings, other factors probably contribute to postoperative clinical outcomes. Also, as shown in preoperative gait analyses, considering the dynamic factors associated with lumbar degenerative kyphosis, a postoperative gait analysis should be used to assess postoperative clinical outcomes. This deserves further study.

BMI (weight $[\mathrm{kg}] /$ height $[\mathrm{m}]^{2}$ ) had a significant correlation with clinical outcomes. Normal patients were defined as those having a BMI of 20-24; overweight patients were those having a BMI of 25-29; obese patients were defined as those having a BMI of $>30$ [19]. In the group where clinical outcomes were good, mean BMI was 23.7 (similar to normal findings); in the group that had poor clinical outcomes, BMI was 26.4 and they were defined as being overweight. In lumbar degenerative kyphosis patients, the extensor muscles in the waist become fragile, kyphosis of the lumbar vertebral spine is reduced and stooping occurs in such a manner that the body is persistently bent in the forward direction. In these cases, with the main focus being on the hip joint-pelvis-sacral vertebrae-lumbar spine in proportion to the weight of the body trunk and upper extremities, the anterior rotational moment is formed. Accordingly, in overweight patients, a greater proportion of rotational loading is exerted in standing or ambulatory positions.

Using T2-weighted axial images of MRI scans, the degree of atrophy of lumbar extensor muscles was measured based on the area of paravertebral muscles and the fat-muscle ratio in the paravertebral muscles. There was no significant difference between the two groups. According to Lee et al. [3], in patients with lumbar degenerative kyphosis, the measurement of the area of paravertebral muscles and the degree of fatty degeneration is the only method. Muscle degeneration in the patient group should be confirmed. But lumbar degenerative kyphosis originates from degeneration of lumbar extensor muscles. In treatment of long level spinal surgery, the degree of muscle injury varies depending on the type of surgery. Owing to this, it is assumed that consistent clinical outcomes cannot be predicted based on measurements acquired during preoperative MRI scans.

Clinical results were found to be satisfactory in $64 \%$ of cases. This compared to a degree of patient satisfaction of $>$ $80 \%$ seen for general cases of degenerative spinal diseases. Because most patients are elderly, there is no great difference in the activity preoperatively vs. postoperatively. Also, based on the characteristics of individual patients, there is a variable degree of expectation of pain, activity, cosmesis and patient satisfaction. A greater difference in these also appeared during postoperative assessment. More attention should be paid to the prediction of clinical outcomes and the determination of who should undergo surgery.

\section{Conclusions}

There is no significant correlation between the preoperative degree of kyphotic deformity and clinical results. On gait analysis, rather than the degree of anterior pelvic tilt being the most important factor, posterior pelvic tilt, compensatory mechanisms of the pelvis and BMI were found to be more important. Because the degree of satisfaction with clinical outcomes and increased activity levels were not relatively higher, a more serious determination of indications for surgery should be made for patients with lumbar degenerative kyphosis. 


\section{REFERENCES}

1. Farcy JP, Schwab FJ. Management of flatback and related kyphotic decompensation syndromes. Spine (Phila $\mathrm{Pa}$ 1976) 1997;22:2452-7.

2. Berven S, Deviren V, Demir-Deviren S, Hu SS, Bradford DS. Studies in the modified Scoliosis Research Society Outcomes Instrument in adults: validation, reliability, and discriminatory capacity. Spine (Phila Pa 1976) 2003;28: 2164-9.

3. Lee JC, Cha JG, Kim Y, Kim YI, Shin BJ. Quantitative analysis of back muscle degeneration in the patients with the degenerative lumbar flat back using a digital image analysis: comparison with the normal controls. Spine (Phila Pa 1976) 2008;33:318-25.

4. Lee CS, Chung SS, Chung KH, Kim SR. Significance of pelvic incidence in the development of abnormal sagittal alignment. J Korean Orthop Assoc 2006;41:274-80.

5. Takemitsu Y, Harada Y, Iwahara T, Miyamoto M, Miyatake Y. Lumbar degenerative kyphosis: clinical, radiological and epidemiological studies. Spine (Phila Pa 1976) 1988;13:1317-26.

6. Kim WJ, Kang JW, Kim KH, et al. Analysis of correction loss after pedicle subtraction osteotomy in patients with sagittal imbalance: radiologic aspects. J Korean Orthop Assoc 2004;39:629-35.

7. Kim WJ, Kang JW, Park JG, et al. Surgical outcome of degenerative lumbar spinal stenosis in patients over 70 years old: a comparative analysis according to surgical method. J Korean Soc Spine Surg 2005;12:101-5.

8. Kim WJ, Kang JW, Yeom JS, et al. The results of pedicle subtraction osteotomy in Iatrogenic flat back syndrome. J Korean Orthop Assoc 2003;38:601-6.

9. Kim WJ, Kang JW, Yang DS, et al. Radiologic analysis of postoperative sagittal plane correction in Lumbar Degenerative Kyphosis (LDK). J Korean Soc Spine Surg 2009;16:177-85.
10. Tsuchiya K, Bridwell KH, Kuklo TR, Lenke LG, Baldus C. Minimum 5-year analysis of L5-S1 fusion using sacropelvic fixation (bilateral $\mathrm{S} 1$ and iliac screws) for spinal deformity. Spine (Phila Pa 1976) 2006;31:303-8.

11. Kim YJ, Bridwell KH, Lenke LG, Rhim S, Cheh G. Pseudarthrosis in long adult spinal deformity instrumentation and fusion to the sacrum: prevalence and risk factor analysis of 144 cases. Spine (Phila Pa 1976) 2006;31:232936.

12. Takemitsu Y, Harada Y, Iwahara T. Low back pain and aging change of spine in Japanese farmers aged more than 40 years. J Jpn Orthop Assoc 1984;58:551-2.

13. Kim WJ, Kang JW, Yeom JS, et al. A comparative analysis of sagittal spinal balance in 100 asymptomatic young and older aged volunteers. J Korean Soc Spine Surg 2003;10:327-34.

14. Andersson BJ, Ortengren R. Myoelectric back muscle activity during sitting. Scand J Rehabil Med Suppl 1974;3:73-90

15. Kim EH, Han SK, Kim HJ. A clinical analysis of surgical treatment of lumbar degenerative kyphosis. J Korean Soc Spine Surg 2001;8:210-8.

16. Lee CS, Kim YT, Kim E. Clinical study of lumbar degenerative kyphosis. J Korean Soc Spine Surg 1997;4:27-35

17. Kim WJ, Kang JW, Kim HY, et al. Change of pelvic tilt before and after gait in patients with lumbar degenerative kyphosis. J Korean Soc Spine Surg 2009;16:95-103.

18. Lee CS, Lee CK, Kim YT, Hong YM, Yoo JH. Dynamic sagittal imbalance of the spine in degenerative flat back: significance of pelvic tilt in surgical treatment. Spine (Phila Pa 1976) 2001;26:2029-35.

19. Himes JH, Dietz WH. Guidelines for overweight in adolescent preventive services: recommendations from an expert committee: the Expert Committee on Clinical Guidelines for Overweight in Adolescent Preventive Services. Am J Clin Nutr 1994;59:307-16. 